\title{
SCIENTIFIC REPORTS

\section{Brain surgery in combination with tyrosine kinase inhibitor and whole brain radiotherapy for epidermal growth factor receptor-mutant non-small-cell lung cancer with brain metastases}

\author{
Hsin-Hua Lee ${ }^{1,2}$, Chien-Hung Chen ${ }^{3}$, Hung-Yi Chuang $\mathbb{1}^{4,5}$, Yu-Wei Huang ${ }^{6} \&$ \\ Ming-Yii Huang ${ }^{1,2,7,8,9 *}$
}

The role of brain surgery (BS) on the survival of patients with non-small-cell lung cancer (NSCLC) and brain metastases (BM), particularly those with epidermal growth factor receptor (EGFR) mutations under tyrosine kinase inhibitors (TKIs) is yet to be defined. We aimed to investigate whether BS could improve the survival of patients in addition to the combination of TKIs and whole brain radiotherapy (WBRT). A cohort of 1394 NSCLC patients between 2011 and 2016 was retrospectively studied. One hundred patients with BM receiving TKI + RT were enrolled. Forty patients $(40 \%)$ received $\mathrm{TKI}+\mathrm{BS}+\mathrm{RT}$, and 60 patients $(60 \%)$ received TKI + RT. Survival time was calculated from the date of BM diagnoses to the date of death or last follow-up. With a median follow-up of 25.6 months $(95 \% \mathrm{Cl}$, 18.6-35.7), the median survival after BM was 18.2 months $(95 \% \mathrm{Cl}, 10.8$ to 27.4$)$ in the TKI+ BS + RT group and 11.8 months $(95 \% \mathrm{Cl}, 5.2$ to 18$)$ in the TKI + RT group. Cox proportional hazards regression model for the patients with the largest BM over $1 \mathrm{~cm}$ showed that TKI + BS + RT group was associated with improved survival relative to TKI $+\mathrm{RT}$ group $(\mathrm{HR}, 0.49 ; 95 \% \mathrm{Cl}, 0.29$ to $0.83 ; P=0.008)$. BS adds significant survival benefits in addition to TKIs and WBRT, especially for patients with EGFR-mutant NSCLC and the largest BM over $1 \mathrm{~cm}$.

Thirty years ago, the median overall survival (OS) after a diagnosis of brain metastasis (BM) for patients with lung adenocarcinoma was 73 days $^{1}$. Lung cancer remains lethal in all nations: 5 -year OS is below $20 \%$ everywhere in Europe, in the range 15-19\% in North America, and as low as 7-9\% in Mongolia and Thailand ${ }^{2}$. The latest U.S. study utilized the National Cancer Data Base to identify 457481 patients with non-small-cell lung cancer (NSCLC) diagnosed between 2010 and 2012. The median and 1-, 2-, and 3-year OS for these patients with BM were 6 months and $29.9 \%, 14.3 \%$, and $8.4 \%$ respectively ${ }^{3}$.

In the epoch of target therapies, screening for specific mutations to guide treatment is necessary. The predictive factors for epidermal growth factor receptor (EGFR) mutations are the female sex, never-smoker status,

\footnotetext{
${ }^{1}$ Program in Environmental and Occupational Medicine, Kaohsiung Medical University and National Health Research Institutes, Kaohsiung, Taiwan. ${ }^{2}$ Department of Radiation Oncology, Kaohsiung Medical University Hospital, Kaohsiung, Taiwan. ${ }^{3}$ Department of Radiation Oncology, Kaohsiung Municipal Ta-Tung Hospital, Kaohsiung, Taiwan. ${ }^{4}$ Department of Occupational and Environmental Medicine, Kaohsiung Medical University Hospital, Kaohsiung Medical University, Kaohsiung, Taiwan. ${ }^{5}$ Faculty of Department of Public Health, College of Health Science, Kaohsiung Medical University, Kaohsiung, Taiwan. ${ }^{6}$ Department of Radiation Oncology, Kaohsiung Chang Gung Memorial Hospital, Kaohsiung, Taiwan. ${ }^{7}$ Department of Radiation Oncology, Faculty of Medicine, College of Medicine, Kaohsiung Medical University, Kaohsiung, Taiwan. ${ }^{8}$ Drug Development and Value Creation Research Center, Kaohsiung Medical University, Kaohsiung, Taiwan. ${ }^{9}$ Center for Cancer Research, Kaohsiung Medical University, Kaohsiung, Taiwan. *email: miyihu@gmail.com
} 
adenocarcinoma histology, and East Asian racial origin ${ }^{4,5}$. However, a U.S. study of 2142 patients with stage I to IV NSCLC found that EGFR mutations in tumors from ever smokers represented $40 \%$ of all mutations detected and those from men represented $31 \%{ }^{6}$. A prospective study of 1482 patients confirmed the EGFR mutation frequency of $51.4 \%$ overall in tumors from Asian patients with adenocarcinoma ${ }^{7}$. Now it is mandatory to detect EGFR mutation prior to treatment. EGFR tyrosine kinase inhibitors (TKIs) have shown a response rate of 70-80\% with improved progression-free survival (PFS) and OS than those obtained with standard chemotherapy in patients harboring EGFR mutations ${ }^{8}$. The use of any first- or second-generation EGFR-TKIs alone for the treatment of intracranial involvement in patients with EGFR mutant-positive lung adenocarcinoma showed a favorable cerebral response rate of more than $50 \%{ }^{9,10}$.

The optimal treatment of BM is debatable. Classic therapeutic options include local therapies such as whole-brain radiation therapy (WBRT), stereotactic radiosurgery and surgical resection, EGFR-TKIs, and chemotherapy. Systemic chemotherapy is considered futile in the treatment of intracranial involvement due to the blood-brain barrier (BBB), which includes efflux pumps on brain capillaries ${ }^{11}$. With the evolution of sophisticated radiation technology, most clinicians integrate radiotherapy (RT) into the comprehensive treatment of patients under TKIs in order to maximize the therapeutic effects ${ }^{12,13}$. The life expectancy in EGFR-mutant patients has been significantly prolonged ${ }^{14-16}$. Because of an aging population and advances in the treatment of NSCLC, patients are living longer and are more likely to experience distant metastases. A recent Taiwanese study reported that EGFR mutation was a predictor for subsequent $\mathrm{BM}^{17}$. Patients with EGFR-mutated NSCLC have a higher cumulative incidence of $\mathrm{BM}^{18}$. They can be surgical candidates because they have already demonstrated the proclivity to longevity despite their cancer diagnoses.

Some may consider novel targeted agents (TKIs: gefitinib, erlotinib, afatinib, icotinib, and osimertinib) are potential alternatives to surgical resection since the survival benefit of surgery seems limited to the subgroup of patients with controlled systemic disease and good performance status ${ }^{19}$. However, improvement in neurosurgical techniques such as microneurosurgery, use of neuronavigation, intraoperative imaging, and cortical and subcortical mapping, along with concurrent progress in neuroanesthesia, has substantially decreased surgical morbidity and mortality ${ }^{20}$. Surgical resection remains an important tool for treating BM from NSCLC, particularly in patients with one large or symptomatic lesion ${ }^{21}$. Patients who received TKIs after a diagnosis of stage IIIB or IV lung cancer and WBRT were reimbursed by the Bureau of National Health Insurance of Taiwan ${ }^{22}$. Herein, we analyzed the factors affecting the prognosis for the patients with EGFR-mutant NSCLC BM under both TKIs and WBRT. The efficacy of brain surgery (BS) will be scrutinized in this research article.

\section{Methods}

Ethics approval statement. The present study (KMUHIRB-E(II)-20180185) was approved and conducted under compliance of the Institutional Review Board (IRB) regulations of Kaohsiung Medical University Hospital. All patients provided written informed consent prior to RT and/or BS. Patient information was anonymized and de-identified before analysis. All data were analyzed anonymously and retrospectively.

Patients and treatment. We sorted 1394 NSCLC patients in the data base of a tertiary university hospital and retrospectively recruited one hundred consecutive patients with pathologically proven lung adenocarcinoma who had received both WBRT and TKIs between January 1, 2011 and June 14, 2016. Their BM was diagnosed by either brain imaging or cytology. The inclusion criteria were positive EGFR mutations, the diagnosis of BM, the use of TKIs, and WBRT. The exclusion criteria were a history of malignancies other than lung cancer, prior brain irradiation, or EGFR-TKI resistance mutation, or incomplete WBRT.

All the patients underwent pretreatment workups comprising a physical examination, a history review, chest radiography, bronchoscopy with a tumor biopsy, chest computed tomography (CT), brain magnetic resonance imaging (MRI) or CT, and routine laboratory studies. The tumor stage was classified according to the seventh edition of the American Joint Committee on Cancer (AJCC) Cancer Staging Manual and Handbook ${ }^{23}$. All patients started taking EGFR-TKIs once the diagnosis of stage IIIB or IV lung cancer with EGFR mutation was established. Some patients received BS which was performed prior to WBRT. BS was recommended at the discretion of neuro-surgeons after discussion with each patient. All patients with or without BS had WBRT. For WBRT, three-dimensional conventional radiotherapy was done by a $2100 \mathrm{C} / \mathrm{D}$ linear accelerator (Varian Medical Systems, Palo Alto, CA). Boost plans were generated by intensity-modulated radiotherapy either with an Eclipse, version 8.6 (Varian Medical Systems Inc., Palo Alto, USA) or Hi-Art helical tomotherapy unit, version 2.2.4.1 (TomoTherapy, Inc., Madison, WI). Our RT schedule was $30 \mathrm{~Gy}$ in 10 fractions or $37.5 \mathrm{~Gy}$ in 15 fractions. Some patients had a boost to BM of $45 \mathrm{~Gy}$ in 10 fractions, or $45 \mathrm{~Gy}$ in 15 fractions. The decision whether to boost BM was made after discussion with each patient.

The following variables were collected: age, sex, stage, initial clinical Tumor and Nodal classification, extracranial metastases, histological grading, smoking history, EGFR mutation, Eastern Cooperative Oncology Group (ECOG) performance status at the time of BM, number of BM, size of largest BM, whether the patient was symptomatic from BM, mean dose of ionizing radiation delivered, name of EGFR-TKI, number of lines of TKI, mean duration of TKI use, and number of lines of chemotherapy. The date of initial cancer diagnosis, the date of BM diagnosis, RT treatments, systemic therapy, most recent follow-up, and death were documented. In addition, a disease-specific Graded Prognostic Assessment (ds-GPA) was calculated for each patient to determine whether the cohorts shared similar prognostic features ${ }^{24}$.

Statistical analysis. The primary end point was the survival after a diagnosis of BM was established. We calculated the survival from the date of BM diagnoses to the date of death from any cause or until the date of the last follow-up. And then we assessed the survival after a diagnosis of BM by Kaplan-Meier methods and used the log-rank test to compare time-to-event distributions. We stratified the data set and compared outcomes by 


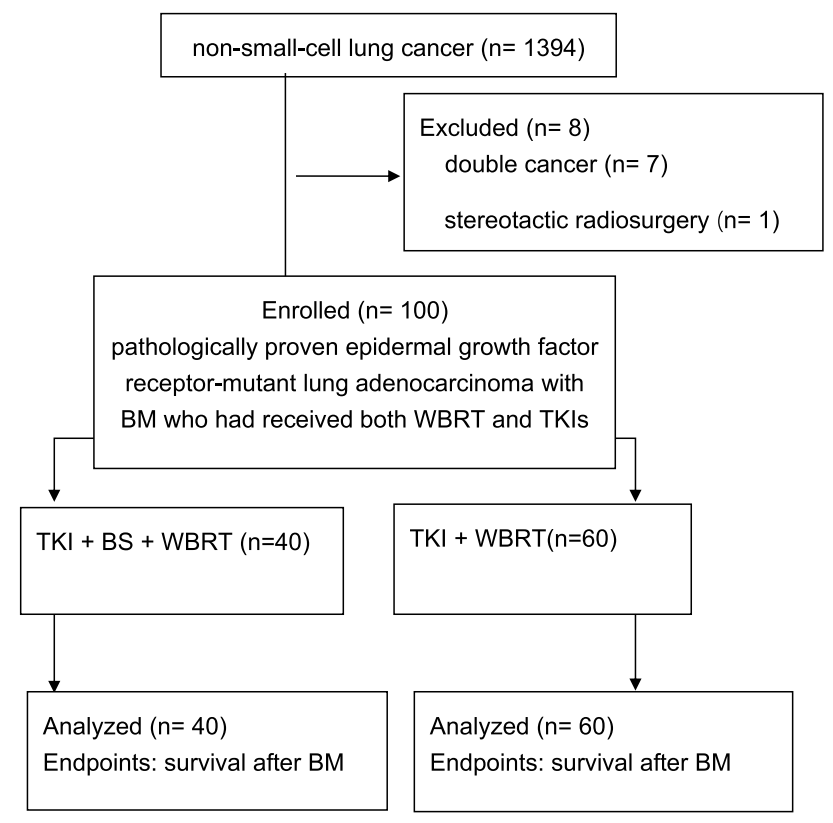

Figure 1. Patient enrollment flow diagram. Survival time was calculated from the date of BM diagnoses to the date of death or last follow-up. Abbreviations: BM: brain metastasis; WBRT: whole brain radiation therapy; TKI: tyrosine kinase inhibitor; BS: brain surgery.

$t$-test or chi-squared test. Besides, we performed univariate analyses and a multivariate Cox proportional hazards regression to examine all collected variables. We calculated the estimated risks of death using hazard ratios (HR) with 95\% confidence intervals (CIs). The level of statistical significance was set at $P<0.05$; all reported $P$ values were two-tailed. The analyses used the SPSS software package, version 19.0 for Windows (SPSS, Chicago, IL, USA).

\section{Results}

There were 147 patients with EGFR-mutant NSCLC and BM, regardless of the treatment. One hundred patients out of 1394 patients in the lung cancer data base were identified after applying the aforementioned inclusion and exclusion criteria (Fig. 1). The clinical characteristics, divided by whether they had BS (TKI + BS + RT group vs TKI + RT group) were sum up in Table 1 . All patients had both EGFR-TKI and WBRT. The mean age of this retrospective cohort was $60 \pm 10$ years \pm standard deviation (SD), 96 patients $(96 \%)$ had an ECOG performance status less than 2 , and $78 \%$ were symptomatic from their BM. Forty patients ( $40 \%)$ received BS (TKI + BS + RT group), and 60 patients $(60 \%)$ did not. Patients who received BS were more likely to have BM larger than $1 \mathrm{~cm}(90 \%$ in the $\mathrm{TKI}+\mathrm{BS}+\mathrm{RT}$ group and $60 \%$ in the TKI + RT group; $P=0.001)$. Patients who received BS were more likely to have EGFR mutation in exon $19(60 \%$ in the TKI + BS + RT group and $36.7 \%$ in the TKI + RT group; $P=0.022)$ and were less likely to have EGFR mutation in exon $21(27.5 \%$ in the TKI + BS + RT group and $48.3 \%$ in the TKI + RT group; $P=0.037)$. One patient has EGFR mutation in both exon 19 and 21 . There was no significant difference in terms of age, gender, stage, initial clinical Tumor and Nodal classification, extracranial metastases, histological grading, smoking history, ECOG performance status at the time of BM, number of BM, whether the patient was symptomatic from BM, mean RT dose, number of lines of TKI, mean duration of TKI use, ds-GPA and number of lines of chemotherapy (all $P>0.05$; Table 1).

All patients started having EGFR-TKI (afatinib, erlotinib, gefitinib or osimertinib) once the diagnosis of stage IIIB or IV lung cancer with EGFR mutation was established. Twelve patients had afatinib; 57 patients had erlotinib; 64 patients had gefitinib; and 5 patients had osimertinib. Thirty-seven (37\%) patients had more than one line of TKIs due to disease progression or intolerance of side effect. The median duration of TKIs use was 14.4 months (95\% CI, 10.7 to 17.9). The median duration of TKIs use was 14 months (95\% CI, 8.3 to 18.3 ) in the TKI + BS + RT group and 14.4 months (95\% CI, 9.6 to 19) in the TKI + RT group. The mean duration of TKIs use were $18 \pm 14$ months and $20 \pm 18.5$ months for patients with and without BS respectively $(P=0.585)$.

After a median follow-up of 25.6 months (95\% CI 18.6 to 35.7), the median survival after BM was 15.1 months (95\% CI, 11.3 to 19.4 ) for the 100 patients in this study. The median survival after BM was 11.2 months $(95 \%$ CI, 8.3 to 14.2) for the 147 patients irrespective of treatment. Specifically, the median survival after BM was 18.2 months (95\% CI, 10.8 to 27.4 ) in the TKI + BS + RT group and 11.8 months (95\% CI, 5.2 to 18) in the TKI + RT group. The mean survival after BM were $21.9 \pm 14.8$ months and $15.6 \pm 14.5$ months for patients with and without BS respectively $(P=0.026)$.

Univariate analysis suggested that BS was a favorable prognostic factors for longer survival (hazard ratio HR, 0.6 ; $95 \%$ CI, 0.38 to $0.95 ; P=0.028$; Fig. 2$)$. In addition, female $(P=0.005)$, exon 19 mutation $(P=0.048)$ and 


\begin{tabular}{|c|c|c|c|c|}
\hline & All & BS & No BS & $P$-value \\
\hline No. of cases & 100 & 40 & 60 & \\
\hline Mean Age (years \pm SD) & $60 \pm 10$ & $61 \pm 9$ & $60 \pm 11$ & 0.643 \\
\hline Sex & & & & 0.799 \\
\hline Female & 64 & 25 & 39 & \\
\hline Male & 36 & 15 & 21 & \\
\hline Initial Clinical stage & & & & 0.775 \\
\hline I-II & 9 & 4 & 5 & \\
\hline III-IV & 91 & 36 & 55 & \\
\hline Initial Tumor classification & & & & 0.78 \\
\hline 1 or 2 & 26 & 11 & 15 & \\
\hline 3 or 4 & 74 & 29 & 45 & \\
\hline Initial Nodal classification & & & & 0.239 \\
\hline 0 or 1 & 38 & 18 & 20 & \\
\hline 2 or 3 & 62 & 22 & 40 & \\
\hline \multicolumn{5}{|l|}{ Extracranial metastases } \\
\hline Bone & 72 & 26 & 46 & 0.203 \\
\hline Lung & 38 & 15 & 23 & 0.933 \\
\hline Liver & 15 & 3 & 12 & 0.086 \\
\hline Histological grade & & & & 0.119 \\
\hline $1-2$ & 40 & 15 & 25 & \\
\hline 3 & 23 & 6 & 17 & \\
\hline NA & 37 & 19 & 18 & \\
\hline \multicolumn{5}{|l|}{ EGFR mutation } \\
\hline Exon 18 & 1 & 0 & 1 & 0.6 (Fisher) \\
\hline Exon 19 & 46 & 24 & 22 & 0.022 \\
\hline Exon 20 & 8 & 5 & 3 & 0.164 (Fisher) \\
\hline Exon 21 & 40 & 11 & 29 & 0.037 \\
\hline NA & 6 & 1 & 5 & 0.397 (Fisher) \\
\hline RT mean boost dose $(\mathrm{cGy} \pm \mathrm{SD})$ & $3779 \pm 748$ & $3908 \pm 612$ & $3694 \pm 821$ & 0.163 \\
\hline dose $>3750$ cGy & 39 & 17 & 22 & \\
\hline dose $\leqq 3750$ cGy & 61 & 23 & 38 & \\
\hline $\begin{array}{l}\text { Number of lines of systemic } \\
\text { chemotherapy }\end{array}$ & & & & 0.518 (Fisher) \\
\hline $0-3$ & 89 & 37 & 52 & \\
\hline$>3$ & 11 & 3 & 8 & \\
\hline \multicolumn{5}{|l|}{ TKI name } \\
\hline afatinib & 12 & 8 & 4 & 0.044 (Fisher) \\
\hline erlotinib & 57 & 22 & 35 & 0.742 \\
\hline gefitinib & 64 & 21 & 43 & 0.05 \\
\hline osimertinib & 5 & 1 & 4 & 0.332 (Fisher) \\
\hline Number of lines of TKI & & & & 0.447 \\
\hline 1 & 63 & 27 & 36 & \\
\hline$>1$ & 37 & 13 & 24 & \\
\hline $\begin{array}{l}\text { Mean TKI use duration } \\
\text { (months } \pm \text { SD) }\end{array}$ & $19.2 \pm 16.8$ & $18 \pm 14$ & $20 \pm 18.5$ & 0.585 \\
\hline ECOG performance status & & & & 0.736 \\
\hline 0 & 52 & 19 & 33 & \\
\hline 1 & 44 & 19 & 25 & \\
\hline 2 & 4 & 2 & 2 & \\
\hline Smoking status & & & & 0.182 \\
\hline Never & 77 & 27 & 15 & \\
\hline Former & 9 & 5 & 4 & \\
\hline Current & 14 & 8 & 6 & \\
\hline Symptomatic BM & & & & 0.168 \\
\hline No & 22 & 6 & 16 & \\
\hline Yes & 78 & 34 & 44 & \\
\hline Size of the largest BM & & & & 0.001 \\
\hline Continued & & & & \\
\hline
\end{tabular}




\begin{tabular}{|l|l|l|l|l|}
\hline & All & BS & No BS & $P$-value \\
\hline$\leqq 1 \mathrm{~cm}$ & 28 & 4 & 24 & \\
\hline$>1 \mathrm{~cm}$ & 72 & 36 & 36 & \\
\hline Number of BM & & & & 0.137 \\
\hline 1 & 18 & 10 & 8 & \\
\hline$>1$ & 82 & 30 & 52 & \\
\hline dsGPA & & & & 0.373 \\
\hline $0.5-1.5$ & 70 & 26 & 44 & \\
\hline $2-4$ & 30 & 14 & 16 & \\
\hline
\end{tabular}

Table 1. Patient and treatment characteristics. Abbreviations: BS: brain surgery; EGFR: epidermal growth factor receptor; RT: radiation therapy; TKI: tyrosine kinase inhibitor; ECOG: Eastern Cooperative Oncology Group; BM: brain metastasis; dsGPA: disease-specific Graded Prognostic Assessment.

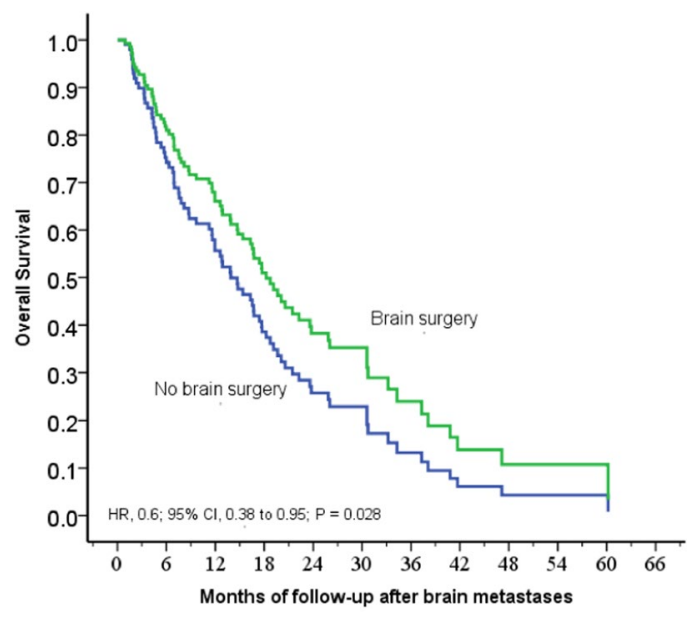

$\begin{array}{llllccccccccc}\text { No. at risk } & & & & & & & & & & & \\ \text { months } & 0 & 6 & 12 & 18 & 24 & 30 & 36 & 42 & 48 & 54 & 60 & 66 \\ \text { Brain surgery } & 40 & 34 & 28 & 21 & 16 & 11 & 7 & 5 & 2 & 2 & 1 & 0 \\ \text { No brain surgery } & 60 & 42 & 29 & 21 & 13 & 9 & 5 & 2 & 1 & 1 & 1 & 1\end{array}$

Figure 2. Cox regression comparing survival after the diagnosis of brain metastasis in epidermal growth factor receptor-mutant non-small-cell lung cancer patients under tyrosine kinase inhibitors treated with and without brain surgery for brain metastases before whole-brain radiation therapy.

single $\mathrm{BM}$ relative to more than $3 \mathrm{BM}(P=0.01)$ were associated with improved OS (Table 1$)$. However, after controlling for significant covariables in a multivariable model including gender, EGFR mutation in exon 19, and number of $\mathrm{BM}$, the TKI + BS + RT group was not associated with improved OS relative to the TKI + RT group (HR, 0.69 ; $95 \%$ CI, 0.43 to 1.128 ; $P=0.134$ ). EGFR mutation in exon 19 was not an independent favorable prognostic factor (HR, $0.83 ; 95 \% \mathrm{CI}, 0.51$ to $1.35 ; P=0.461)$. Table 2 shows two independent favorable prognostic factors including female gender ( $\mathrm{HR}, 0.56 ; 95 \% \mathrm{CI}, 0.34$ to $0.9 ; P=0.017)$ and single $\mathrm{BM}$ relative to more than 3 $\mathrm{BM}(\mathrm{HR}, 2.23 ; 95 \% \mathrm{CI}, 1.11$ to $4.45 ; P=0.024)$.

BM size. In order to identify potential differences in the benefits of BS in patients by the size of largest BM, we selected 72 patients with the largest BM larger than $1 \mathrm{~cm}$. Thirty-six (50\%) patients had BS. Cox regression analysis revealed that BS was a strong favorable prognostic factor for longer survival (HR, 0.5; $95 \% \mathrm{CI}, 0.3$ to $0.84 ; P=0.008$; Fig. 3). In Table 3, after controlling for significant covariables in a multivariable model, the $\mathrm{TKI}+\mathrm{BS}+\mathrm{RT}$ group was associated with improved OS relative to the TKI + RT group (HR, 0.49; 95\% CI, 0.29 to $0.83 ; P=0.008)$. Clinical nodal classification $0-1$ relative to $2-3(\mathrm{HR}, 2.23 ; 95 \% \mathrm{CI}, 1.27$ to $3.92 ; P=0.005)$ and the use of erlotinib (HR, $0.49 ; 95 \% \mathrm{CI}, 0.29$ to $0.85 ; P=0.011)$ were also beneficial.

EGFR mutation. In order to identify potential differences in the benefits of BS in patients with EGFR mutation, we selected 85 patients with mutation in exon 19 or 21 or both. Thirty-four (40\%) patients had BS. Cox regression analysis revealed that female, BS and single BM were favorable prognostic factors for longer survival (Table 4). However, after controlling for significant covariables in a multivariable model, the TKI + BS + RT group was not associated with improved OS relative to the TKI + RT group (HR, 0.66; 95\% CI, 0.4 to $1.11 ; P=0.116$ ). Female (HR, $0.47 ; 95 \% \mathrm{CI}, 0.28$ to $0.78 ; P=0.004)$ and single $\mathrm{BM}$ relative to more than $3 \mathrm{BM}(\mathrm{HR}, 2.41 ; 95 \% \mathrm{CI}$, 1.16 to $5 ; P=0.018$ ) were two independent favorable prognostic factors. 


\begin{tabular}{|c|c|c|c|c|}
\hline & \multicolumn{2}{|l|}{ Univariate analyses } & \multicolumn{2}{|c|}{ Multivariate analyses } \\
\hline & HR $(95 \% \mathrm{CI})$ & $P$-value & HR $(95 \% \mathrm{CI})$ & $P$-value \\
\hline \multicolumn{5}{|l|}{ Age } \\
\hline$>65$ vs. $\leqq 65$ & $1.35(0.83$ to 2.2$)$ & 0.226 & & \\
\hline Female vs. male & $0.5(0.31$ to 0.79$)$ & 0.005 & $0.56(0.34$ to 0.9$)$ & 0.017 \\
\hline \multicolumn{5}{|l|}{ Initial Clinical stage } \\
\hline III-IV vs. I-II & 1.25 (0.54 to 2.88$)$ & 0.6 & & \\
\hline \multicolumn{5}{|c|}{ Initial Tumor classification } \\
\hline III-IV vs. I-II & $1.39(0.83$ to 2.33$)$ & 0.208 & & \\
\hline \multicolumn{5}{|c|}{ Initial Nodal classification } \\
\hline $2-3$ vs. $0-1$ & $1.48(0.93$ to 2.35$)$ & 0.097 & & \\
\hline \multicolumn{5}{|l|}{ EGFR mutation } \\
\hline \multicolumn{5}{|l|}{ Exon 19 or 21} \\
\hline Yes vs. no & $0.69(0.38$ to 1.26$)$ & 0.225 & & \\
\hline \multicolumn{5}{|l|}{ Exon 19} \\
\hline Yes vs. no & $0.63(0.4$ to 1$)$ & 0.048 & $0.83(0.51$ to 1.35$)$ & 0.461 \\
\hline \multicolumn{5}{|l|}{ Exon 20} \\
\hline Yes vs. no & $0.95(0.44$ to 2.08$)$ & 0.905 & & \\
\hline \multicolumn{5}{|l|}{ Exon 21} \\
\hline Yes vs. no & $1.18(0.75$ to 1.85$)$ & 0.47 & & \\
\hline \multicolumn{5}{|l|}{ Brain surgery } \\
\hline Yes vs. no & $0.6(0.38$ to 0.95$)$ & 0.028 & $0.69(0.43$ to 1.12$)$ & 0.134 \\
\hline \multicolumn{5}{|c|}{ RT boost dose $>3750 \mathrm{cGy}$} \\
\hline Yes vs. no & $0.84(0.53$ to 1.32$)$ & 0.441 & & \\
\hline \multicolumn{5}{|c|}{ Number of lines of systemic chemotherapy } \\
\hline$>3$ vs. $0-3$ & $1.26(0.63$ to 2.53$)$ & 0.519 & & \\
\hline \multicolumn{5}{|l|}{ TKI name } \\
\hline \multicolumn{5}{|l|}{ afatinib } \\
\hline Yes vs. no & 0.56 (0.26 to 1.22$)$ & 0.144 & & \\
\hline \multicolumn{5}{|l|}{ erlotinib } \\
\hline Yes vs. no & 0.72 (0.46 to 1.12$)$ & 0.141 & & \\
\hline \multicolumn{5}{|l|}{ gefitinib } \\
\hline Yes vs. no & $1.43(0.89$ to 2.31$)$ & 0.142 & & \\
\hline \multicolumn{5}{|l|}{ osimertinib } \\
\hline Yes vs. no & 0.68 (0.21 to 2.15$)$ & 0.505 & & \\
\hline \multicolumn{5}{|c|}{ Number of lines of TKI } \\
\hline$>1$ vs. 1 & $0.8(0.5$ to 1.26$)$ & 0.326 & & \\
\hline \multicolumn{5}{|c|}{ ECOG performance status } \\
\hline 1 vs. 0 & $1.03(0.66$ to 1.61$)$ & 0.885 & & \\
\hline 2 vs. 0 & $0.46(0.11$ to 1.91$)$ & 0.287 & & \\
\hline Smoking status & & & & \\
\hline $\begin{array}{l}\text { Former or current } \\
\text { vs. never }\end{array}$ & $1.39(0.84$ to 2.3$)$ & 0.206 & & \\
\hline Symptomatic brain $n$ & netastases & & & \\
\hline Yes vs. no & $1.13(0.65$ to 1.95$)$ & 0.671 & & \\
\hline Size of the largest bra & ain tumor & & & \\
\hline$>1 \mathrm{~cm}$ vs. $\leqq 1 \mathrm{~cm}$ & $1.51(0.9$ to 2.53$)$ & 0.121 & & \\
\hline No. of brain metasta & & & & \\
\hline $2-3$ vs. 1 & 2.11 (0.95 to 4.93$)$ & 0.068 & $1.83(0.81$ to 4.14$)$ & 0.149 \\
\hline$>3$ vs. 1 & 2.45 (1.24 to 4.72$)$ & 0.01 & 2.23 (1.11 to 4.45$)$ & 0.024 \\
\hline dsGPA & & & & \\
\hline $0.5-1.5$ vs. $2-4$ & $1.6(0.96$ to 2.65$)$ & 0.07 & & \\
\hline
\end{tabular}

Table 2. Univariate and multivariate Cox regression analyses of covariables associated with survival after brain metastases. Abbreviations: EGFR: epidermal growth factor receptor; RT: radiation therapy; TKI: tyrosine kinase inhibitor; ECOG: Eastern Cooperative Oncology Group; dsGPA: disease-specific Graded Prognostic Assessment. 


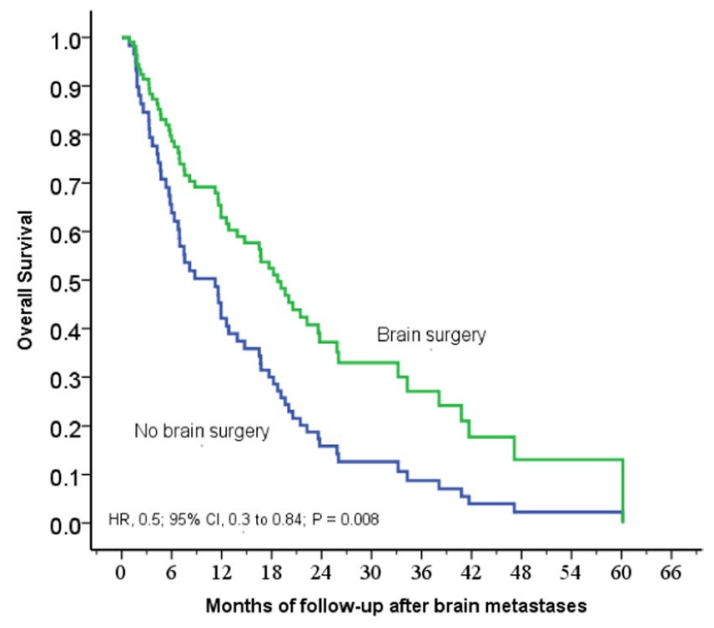

$\begin{array}{llccccccccccc}\text { No. at risk } & & & & & & & & & & & & \\ \text { months } & 0 & 6 & 12 & 18 & 24 & 30 & 36 & 42 & 48 & 54 & 60 & 66 \\ \text { Brain surgery } & 36 & 30 & 24 & 17 & 13 & 9 & 7 & 5 & 2 & 2 & 1 & 0 \\ \text { No brain surgery } & 36 & 22 & 13 & 12 & 5 & 2 & 2 & 1 & 0 & 0 & 0 & 0\end{array}$

Figure 3. Cox regression comparing survival after the diagnosis of brain metastasis in epidermal growth factor receptor-mutant non-small-cell lung cancer patients under tyrosine kinase inhibitors with a size of the largest brain metastasis over $1 \mathrm{~cm}$ treated with and without brain surgery for brain metastases before whole-brain radiation therapy.

\section{Discussion}

The mainstay of treatment for BM consisted of surgical resection, RT, or a combination of these modalities. A prospective randomized study showed that patients with cancer and a single BM who received BS plus RT lived longer ${ }^{25}$. A further study showed that BS followed by consolidative WBRT was better than BS alone for local control $^{26}$. RT is commonly used following BS since local recurrence occurs in more than $50 \%$ of patients ${ }^{27}$. In two randomized trials, postoperative adjuvant WBRT reduced the incidence of local recurrence by half ${ }^{27,28}$. Twenty years ago, additional postoperative WBRT with $30 \mathrm{~Gy}$ for patients with single BM was reported (BS + WBRT: median OS 13 months; BS only: median OS 8 months). In addition, the rate of cerebral recurrence was distinctly higher in the non-WBRT group ${ }^{29}$.

Toffart et al. concluded that the survival of NSCLC with synchronous solitary M1 was more similar to stage III than other stage IV NSCLC and advocated for BS $^{30}$. In this large retrospective study of 4832 patients, $64 \%$ of patients had BM. Operation conducted at both primary and metastatic sites (HR 0.35, 95\% CI: 0.19 to 0.65 ) was an independent prognostic factor for longer survival. For accessible tumors with diameter of more than $3 \mathrm{~cm}$, BS still carries the advantages of obtaining histological diagnosis, providing immediate symptomatic relief by removal of local mass effect and source of edema, and decreasing the length of steroid use ${ }^{21}$.

At the time of the studies of Patchell et al., however, EGFR-TKIs were not available ${ }^{25,28}$. In the era of EGFR mutations and TKIs, we seek to compare survival trends that are likely to be attributable to combined treatment, especially BS plus TKIs and WBRT. We investigated one hundred patients who received TKIs as a first-line therapy for advanced EGFR-mutant NSCLC in a tertiary cancer center. Because the administration of EGFR-TKI has limited penetration across BBB, combined RT would provide better outcome ${ }^{31}$. According to Burel-Vandenbos et al., BM occurring during the course of TKI, despite good control of extracranial disease, is possibly due to insufficient concentration of TKI in cerebral spinal fluid ${ }^{32}$ although raising TKI doses might increase the possibility of drug intolerance. Soon et al. conducted a meta-analysis from 2008 to July 2014, and reported there was a better 2 -year OS (HR 1.33, 95\% CI 1.00-1.77; $P=0.05$ ) for patients with upfront WBRT compared with TKI alone ${ }^{33}$.

However, NSCLC is a radioresistant malignancy and 30 Gy of WBRT may not be sufficient to sterilize the metastatic brain lesions ${ }^{32}$, therefore combination therapy is required. We hypothesized that BS would be beneficial prior to WBRT. After controlling for significant covariables in a multivariable model, EGFR mutation in exon 19 (HR, $0.83 ; 95 \% \mathrm{CI}, 0.51$ to $1.35 ; P=0.461$ ) or the TKI + BS + RT group was not associated with improved survival relative to the TKI $+\mathrm{RT}$ group $(\mathrm{HR}, 0.69 ; 95 \% \mathrm{CI}, 0.43$ to $1.12 ; P=0.134)$. However, for the 72 patients with the largest BM over $1 \mathrm{~cm}$, multivariate analysis showed that the TKI + BS + RT group was associated with improved survival relative to the TKI + RT group (HR, $0.49 ; 95 \% \mathrm{CI}, 0.29$ to $0.83 ; P=0.008)$. For the majority of the patients with EGFR mutation in exon 19 or 21, Female and single BM relative to more than $3 \mathrm{BM}$ were two strong independent favorable prognostic factors.

The present study demonstrated the advantage of BS, especially for patients with stage IV EGFR-mutant NSCLC who had the largest BM over $1 \mathrm{~cm}$. To the best of our knowledge, this is the first study to confirm the advantage from combination therapy of TKI $+\mathrm{BS}+\mathrm{RT}$. Until recently, there was no prospective randomized trial regarding the addition of BS to TKIs and WBRT. 


\begin{tabular}{|c|c|c|c|c|}
\hline & \multicolumn{2}{|l|}{ Univariate analyses } & \multicolumn{2}{|c|}{ Multivariate analyses } \\
\hline & HR $(95 \% \mathrm{CI})$ & $P$-value & HR $(95 \% \mathrm{CI})$ & $P$-value \\
\hline \multicolumn{5}{|l|}{ Age } \\
\hline$>65$ vs. $\leqq 65$ & $1.32(0.77$ to 2.29$)$ & 0.318 & & \\
\hline Female vs. male & $0.59(0.35$ to 1$)$ & 0.052 & & \\
\hline \multicolumn{5}{|l|}{ Initial Clinical stage } \\
\hline III-IV vs. I-II & $1.38(0.5$ to 3.81$)$ & 0.538 & & \\
\hline \multicolumn{5}{|l|}{ Initial Tumor classification } \\
\hline III-IV vs. I-II & $0.92(0.5$ to 1.67$)$ & 0.772 & & \\
\hline \multicolumn{5}{|l|}{ Initial Nodal classification } \\
\hline $2-3$ vs. $0-1$ & 1.67 (0.98 to 2.83$)$ & 0.058 & \begin{tabular}{|l|}
$2.23(1.27$ to \\
$3.92)$
\end{tabular} & 0.005 \\
\hline \multicolumn{5}{|l|}{ EGFR mutation } \\
\hline \multicolumn{5}{|l|}{ Exon 19 or 21} \\
\hline Yes vs. no & $0.815(0.43$ to 1.55$)$ & 0.533 & & \\
\hline \multicolumn{5}{|l|}{ Exon 19} \\
\hline Yes vs. no & $0.68(0.41$ to 1.15$)$ & 0.15 & & \\
\hline \multicolumn{5}{|l|}{ Exon 20} \\
\hline Yes vs. no & $0.82(0.372$ to 1.81$)$ & 0.622 & & \\
\hline \multicolumn{5}{|l|}{ Exon 21} \\
\hline Yes vs. no & $1.12(0.65$ to 1.9$)$ & 0.687 & & \\
\hline \multicolumn{5}{|l|}{ Brain surgery } \\
\hline Yes vs. no & $0.5(0.3$ to 0.84$)$ & 0.008 & \begin{tabular}{|l}
$0.49(0.29$ to \\
$0.83)$
\end{tabular} & 0.008 \\
\hline \multicolumn{5}{|l|}{ RT boost dose $>3750$ cGy } \\
\hline Yes vs. no & $1.1(0.65$ to 1.85$)$ & 0.726 & & \\
\hline \multicolumn{5}{|c|}{ Number of lines of systemic chemotherapy } \\
\hline$>3$ vs. $0-3$ & $1.18(0.53$ to 2.61$)$ & 0.684 & & \\
\hline \multicolumn{5}{|l|}{ TKI name } \\
\hline \multicolumn{5}{|l|}{ afatinib } \\
\hline Yes vs. no & $1.16(0.46$ to 2.9$)$ & 0.757 & & \\
\hline \multicolumn{5}{|l|}{ erlotinib } \\
\hline Yes vs. no & $0.57(0.34$ to 0.95$)$ & 0.031 & \begin{tabular}{|l|}
$0.49(0.29$ to \\
$0.85)$
\end{tabular} & 0.011 \\
\hline \multicolumn{5}{|l|}{ gefitinib } \\
\hline Yes vs. no & $1.43(0.83$ to 2.48$)$ & 0.199 & & \\
\hline \multicolumn{5}{|l|}{ osimertinib } \\
\hline Yes vs. no & $1.31(0.41$ to 4.19$)$ & 0.655 & & \\
\hline \multicolumn{5}{|l|}{ Number of lines of TKI } \\
\hline$>1$ vs. 1 & $0.692(0.4$ to 1.2$)$ & 0.192 & & \\
\hline \multicolumn{5}{|l|}{ ECOG performance status } \\
\hline 1 vs. 0 & $0.96(0.57$ to 1.62$)$ & 0.889 & & \\
\hline 2 vs. 0 & 0.59 (0.14 to 2.47$)$ & 0.467 & & \\
\hline \multicolumn{5}{|l|}{ Smoking status } \\
\hline Former or current vs. never & 1.25 (0.71 to 2.2$)$ & 0.434 & & \\
\hline Symptomatic brain metastase & & & & \\
\hline Yes vs. no & $0.73(0.31$ to 1.71$)$ & 0.471 & & \\
\hline No. of brain metastases & & & & \\
\hline $2-3$ vs. 1 & $1.62(0.75$ to 3.49$)$ & 0.216 & & \\
\hline$>3$ vs. 1 & $1.4(0.58$ to 3.39$)$ & 0.451 & & \\
\hline dsGPA & & & & \\
\hline $0.5-1.5$ vs. $2-4$ & $1.38(0.74$ to 2.55$)$ & 0.311 & & \\
\hline
\end{tabular}

Table 3. For patients with the largest brain metastasis over $1 \mathrm{~cm}$ : univariate and multivariate Cox regression analyses of covariables associated with survival after the diagnosis of brain metastasis. Abbreviations: EGFR: epidermal growth factor receptor; RT: radiation therapy; TKI: tyrosine kinase inhibitor; ECOG: Eastern Cooperative Oncology Group; dsGPA: disease-specific Graded Prognostic Assessment.

The limitations of the current study are the inherent biases in retrospective studies. The pitfalls include limited patient numbers, possible selection bias from surgical intervention, incomplete records of post-operative complications, cognitive evaluation and intracranial control. This study enrolled a real-world population of NSCLC 


\begin{tabular}{|c|c|c|c|c|}
\hline & \multicolumn{2}{|l|}{ Univariate analyses } & \multicolumn{2}{|c|}{ Multivariate analyses } \\
\hline & HR (95\%CI) & $P$-value & HR (95\%CI) & $P$-value \\
\hline \multicolumn{5}{|l|}{ Age } \\
\hline$>65$ vs. $\leqq 65$ & $1.32(0.78$ to 2.24$)$ & 0.302 & & \\
\hline Female vs. male & $0.46(0.28$ to 0.76$)$ & 0.003 & $0.47(0.28$ to 0.78$)$ & 0.004 \\
\hline \multicolumn{5}{|l|}{ Initial Clinical stage } \\
\hline III-IV vs. I-II & $2.57(0.63$ to 10.51$)$ & 0.19 & & \\
\hline \multicolumn{5}{|l|}{ Initial Tumor classification } \\
\hline III-IV vs. I-II & $1.43(0.81$ to 2.51$)$ & 0.215 & & \\
\hline \multicolumn{5}{|l|}{ Initial Nodal classification } \\
\hline $2-3$ vs. $0-1$ & $1.53(0.91$ to 2.56$)$ & 0.105 & & \\
\hline \multicolumn{5}{|l|}{ Brain surgery } \\
\hline Yes vs. no & $0.59(0.36$ to 0.97$)$ & 0.036 & $0.66(0.4$ to 1.11$)$ & 0.116 \\
\hline \multicolumn{5}{|l|}{ RT boost dose > 3750cGy } \\
\hline Yes vs. no & $0.81(0.48$ to 1.35$)$ & 0.41 & & \\
\hline \multicolumn{5}{|c|}{ Number of lines of systemic chemotherapy } \\
\hline$>3$ vs. $0-3$ & $1.96(0.93$ to 4.15$)$ & 0.077 & & \\
\hline \multicolumn{5}{|l|}{ TKI name } \\
\hline \multicolumn{5}{|l|}{ afatinib } \\
\hline Yes vs. no & $0.45(0.18$ to 1.12$)$ & 0.86 & & \\
\hline \multicolumn{5}{|l|}{ erlotinib } \\
\hline Yes vs. no & $0.82(0.51$ to 1.33$)$ & 0.427 & & \\
\hline \multicolumn{5}{|l|}{ gefitinib } \\
\hline Yes vs. no & $1.49(0.86$ to 2.6$)$ & 0.159 & & \\
\hline \multicolumn{5}{|l|}{ osimertinib } \\
\hline Yes vs. no & $0.65(0.16$ to 2.65$)$ & 0.543 & & \\
\hline \multicolumn{5}{|l|}{ Number of lines of TKI } \\
\hline$>1$ vs. 1 & $0.938(0.57$ to 1.54$)$ & 0.798 & & \\
\hline \multicolumn{5}{|l|}{ ECOG performance status } \\
\hline 1 vs. 0 & $1.05(0.65$ to 1.71$)$ & 0.842 & & \\
\hline 2 vs. 0 & $0.34(0.05$ to 2.46$)$ & 0.283 & & \\
\hline \multicolumn{5}{|l|}{ Smoking status } \\
\hline Former or current vs. never & $1.43(0.81$ to 2.54$)$ & 0.216 & & \\
\hline \multicolumn{5}{|l|}{ Symptomatic brain metastases } \\
\hline Yes vs. no & $1.13(0.61$ to 2.07$)$ & 0.697 & & \\
\hline \multicolumn{5}{|l|}{ Size of largest brain tumor } \\
\hline$>1 \mathrm{~cm} \mathrm{vs} \leqq 1 \mathrm{~cm}$ & $1.47(0.85$ to 2.53$)$ & 0.168 & & \\
\hline \multicolumn{5}{|l|}{ No. of brain metastases } \\
\hline $2-3$ vs. 1 & $2.03(0.84$ to 4.91$)$ & 0.118 & $1.8(0.74$ to 4.4$)$ & 0.198 \\
\hline$>3$ vs. 1 & $2.57(1.25$ to 5.28$)$ & 0.01 & $2.41(1.16$ to 5$)$ & 0.018 \\
\hline \multicolumn{5}{|l|}{ dsGPA } \\
\hline $0.5-1.5$ vs. $2-4$ & $1.58(0.92$ to 2.71$)$ & 0.098 & & \\
\hline
\end{tabular}

Table 4. For patients with EGFR mutation in exon 19 or exon 21: univariate and multivariate Cox regression analyses of covariables associated with survival after the diagnosis of brain metastasis. Abbreviations: EGFR: epidermal growth factor receptor; RT: radiation therapy; TKI: tyrosine kinase inhibitor; ECOG: Eastern Cooperative Oncology Group; dsGPA: disease-specific Graded Prognostic Assessment.

patients, including sicker patients who were not eligible for a clinical trial. Although the patients were not randomized, fundamentally, similar characteristics existed between TKI + BS + RT and TKI + RT groups. In the current study, we focused on prognostic features, molecular markers, and survival change from the addition of BS to the combination of TKIs and WBRT.

\section{Conclusions}

BS prior to WBRT adds significant survival benefits in addition to the combination of TKIs and WBRT, especially for patients with EGFR-mutant NSCLC who had the largest BM over $1 \mathrm{~cm}$. This observational study on BS outcome needs to be interpreted with some caution because there are potential confounding factors. The result should be cautiously applied. Further prospective study is warranted. 


\section{Data availability}

The data used to support the findings of this study are included within the article.

Received: 28 February 2019; Accepted: 1 November 2019;

Published online: 14 November 2019

\section{References}

1. Sorensen, J. B., Hansen, H. H., Hansen, M. \& Dombernowsky, P. Brain metastases in adenocarcinoma of the lung: frequency, risk groups, and prognosis. Journal of clinical oncology: official journal of the American Society of Clinical Oncology 6, 1474-1480, https:// doi.org/10.1200/JCO.1988.6.9.1474 (1988).

2. Allemani, C. et al. Global surveillance of cancer survival 1995-2009: analysis of individual data for 25,676,887 patients from 279 population-based registries in 67 countries (CONCORD-2). Lancet (London, England) 385, 977-1010, https://doi.org/10.1016/ S0140-6736(14)62038-9 (2015).

3. Waqar, S. N. et al. Non-small-cell Lung Cancer With Brain Metastasis at Presentation. Clinical lung cancer 19, e373-e379, https:// doi.org/10.1016/j.cllc.2018.01.007 (2018).

4. Rosell, R. et al. Screening for epidermal growth factor receptor mutations in lung cancer. The New England journal of medicine 361, 958-967, https://doi.org/10.1056/NEJMoa0904554 (2009).

5. Tanaka, T. et al. Frequency of and variables associated with the EGFR mutation and its subtypes. International journal of cancer 126, 651-655, https://doi.org/10.1002/ijc.24746 (2010).

6. D'Angelo, S. P. et al. Incidence of EGFR exon 19 deletions and L858R in tumor specimens from men and cigarette smokers with lung adenocarcinomas. Journal of clinical oncology: official journal of the American Society of Clinical Oncology 29, 2066-2070, https://doi. org/10.1200/JCO.2010.32.6181 (2011)

7. Shi, Y. et al. A prospective, molecular epidemiology study of EGFR mutations in Asian patients with advanced non-small-cell lung cancer of adenocarcinoma histology (PIONEER). Journal of thoracic oncology: official publication of the International Association for the Study of Lung Cancer 9, 154-162, https://doi.org/10.1097/JTO.0000000000000033 (2014).

8. D'Antonio, C. et al. Bone and brain metastasis in lung cancer: recent advances in therapeutic strategies. Therapeutic advances in medical oncology 6, 101-114, https://doi.org/10.1177/1758834014521110 (2014).

9. Li, M. X. et al. Central nervous system progression in advanced non-small cell lung cancer patients with EGFR mutations in response to first-line treatment with two EGFR-TKIs, gefitinib and erlotinib: a comparative study. BMC cancer 17, 245, https://doi. org/10.1186/s12885-017-3165-0 (2017).

10. Schuler, M. et al. First-Line Afatinib versus Chemotherapy in Patients with Non-Small Cell Lung Cancer and Common Epidermal Growth Factor Receptor Gene Mutations and Brain Metastases. Journal of thoracic oncology: official publication of the International Association for the Study of Lung Cancer 11, 380-390, https://doi.org/10.1016/j.jtho.2015.11.014 (2016).

11. Di Lorenzo, R. \& Ahluwalia, M. S. Targeted therapy of brain metastases: latest evidence and clinical implications. Therapeutic advances in medical oncology $9,781-796$, https://doi.org/10.1177/1758834017736252 (2017)

12. Trifiletti, D. M. et al. National trends in radiotherapy for brain metastases at time of diagnosis of non-small cell lung cancer. Journal of clinical neuroscience: official journal of the Neurosurgical Society of Australasia 45, 48-53, https://doi.org/10.1016/j. jocn.2017.08.028 (2017).

13. Shukuya, T. et al. Continuous EGFR-TKI administration following radiotherapy for non-small cell lung cancer patients with isolated CNS failure. Lung cancer (Amsterdam, Netherlands) 74, 457-461, https://doi.org/10.1016/j.lungcan.2011.04.007 (2011).

14. Xia, B., Zhang, S. \& Ma, S. Management of non-small cell lung cancer with EGFR mutation: the role of radiotherapy in the era of tyrosine kinase inhibitor therapy-opportunities and challenges. Journal of thoracic disease $9,3385-3393$, https://doi.org/10.21037/ jtd.2017.09.67 (2017).

15. O'Kane, G. M. \& Leighl, N. B. Systemic Therapy of Lung Cancer CNS Metastases Using Molecularly Targeted Agents and Immune Checkpoint Inhibitors. CNS drugs 32, 527-542, https://doi.org/10.1007/s40263-018-0526-4 (2018).

16. Yoshida, T. et al. RECIST progression patterns during EGFR tyrosine kinase inhibitor treatment of advanced non-small cell lung cancer patients harboring an EGFR mutation. Lung cancer (Amsterdam, Netherlands) 90, 477-483, https://doi.org/10.1016/j. lungcan.2015.09.025 (2015).

17. Chang, W. Y. et al. The impact of EGFR mutations on the incidence and survival of stages I to III NSCLC patients with subsequent brain metastasis. PloS one 13, e0192161, https://doi.org/10.1371/journal.pone.0192161 (2018).

18. Vaca, S. D., Connolly, I. D., Ho, C., Neal, J. \& Hayden Gephart, M. Commentary: Treatment Considerations for Patients With Epidermal Growth Factor Receptor-Mutated Non-Small Cell Lung Cancer Brain Metastases in the Era of Tyrosine Kinase Inhibitors. Neurosurgery 82, E6-E14, https://doi.org/10.1093/neuros/nyx429 (2018).

19. Franchino, F., Ruda, R. \& Soffietti, R. Mechanisms and Therapy for Cancer Metastasis to the Brain. Frontiers in oncology 8, 161, https://doi.org/10.3389/fonc.2018.00161 (2018).

20. Sawaya, R. et al. Neurosurgical outcomes in a modern series of 400 craniotomies for treatment of parenchymal tumors. Neurosurgery 42, 1044-1055; discussion 1055-1046 (1998).

21. Goldberg, S. B., Contessa, J. N., Omay, S. B. \& Chiang, V. Lung Cancer Brain Metastases. Cancer J 21, 398-403, https://doi. org/10.1097/PPO.0000000000000146 (2015).

22. Yao, Z. H. et al. Real-World Data on Prognostic Factors for Overall Survival in EGFR Mutation-Positive Advanced Non-Small Cell Lung Cancer Patients Treated with First-Line Gefitinib. The oncologist 22, 1075-1083, https://doi.org/10.1634/ theoncologist.2016-0331 (2017).

23. Edge, S. B. et al. AJCC Cancer Staging Manual. 7th ed. (Springer-Verlag, 2010).

24. Sperduto, P. W. et al. Diagnosis-specific prognostic factors, indexes, and treatment outcomes for patients with newly diagnosed brain metastases: a multi-institutional analysis of 4,259 patients. International journal of radiation oncology, biology, physics 77, 655-661, https://doi.org/10.1016/j.ijrobp.2009.08.025 (2010).

25. Patchell, R. A. et al. A randomized trial of surgery in the treatment of single metastases to the brain. The New England journal of medicine 322, 494-500, https://doi.org/10.1056/NEJM199002223220802 (1990).

26. Kalkanis, S. N. et al. The role of surgical resection in the management of newly diagnosed brain metastases: a systematic review and evidence-based clinical practice guideline. Journal of neuro-oncology 96, 33-43, https://doi.org/10.1007/s1 1060-009-0061-8 (2010).

27. Kocher, M. et al. Adjuvant whole-brain radiotherapy versus observation after radiosurgery or surgical resection of one to three cerebral metastases: results of the EORTC 22952-26001 study. Journal of clinical oncology: official journal of the American Society of Clinical Oncology 29, 134-141, https://doi.org/10.1200/JCO.2010.30.1655 (2011).

28. Patchell, R. A. et al. Postoperative radiotherapy in the treatment of single metastases to the brain: a randomized trial. Jama 280, 1485-1489 (1998).

29. Schackert, G., Steinmetz, A., Meier, U. \& Sobottka, S. B. Surgical management of single and multiple brain metastases: results of a retrospective study. Onkologie 24, 246-255, https://doi.org/10.1159/000055087 (2001).

30. Toffart, A. C. et al. Operation and Chemotherapy: Prognostic Factors for Lung Cancer With One Synchronous Metastasis. The Annals of thoracic surgery 105, 957-965, https://doi.org/10.1016/j.athoracsur.2017.10.040 (2018). 
31. Zhang, J., Yu, J., Sun, X. \& Meng, X. Epidermal growth factor receptor tyrosine kinase inhibitors in the treatment of central nerve system metastases from non-small cell lung cancer. Cancer letters 351, 6-12, https://doi.org/10.1016/j.canlet.2014.04.019 (2014).

32. Burel-Vandenbos, F., Ambrosetti, D., Coutts, M. \& Pedeutour, F. EGFR mutation status in brain metastases of non-small cell lung carcinoma. Journal of neuro-oncology 111, 1-10, https://doi.org/10.1007/s11060-012-0990-5 (2013).

33. Soon, Y. Y., Leong, C. N., Koh, W. Y. \& Tham, I. W. EGFR tyrosine kinase inhibitors versus cranial radiation therapy for EGFR mutant non-small cell lung cancer with brain metastases: a systematic review and meta-analysis. Radiotherapy and oncology: journal of the European Society for Therapeutic Radiology and Oncology 114, 167-172, https://doi.org/10.1016/j.radonc.2014.12.011 (2015).

\section{Acknowledgements}

This work was supported financially by grants from Kaohsiung Medical University Hospital (KMUH107-7G11), the Drug Development and Value Creation Research Center, Kaohsiung Medical University (KMU-TC108A03) and Center for Cancer Research, Kaohsiung Medical University (KMU-TC108A04), the Ministry of Science and Technology [MOST 107-2922-I-037-015; MOST 108-2314-B-037-021-MY3] in Taiwan. The funding source had no role in the study design; in the collection, analysis and interpretation of data; in the writing of the report and in the decision to submit the article for publication.

\section{Author contributions}

H.H.L., C.H.C. and M.Y.H. participated in the design of the study, interpretation of data, and drafted the manuscript. H.H.L. wrote the manuscript. C.H.C., Y.W.H. and H.Y.C. performed the statistical analysis and interpreted the clinical data. All authors read and approved the final manuscript.

\section{Competing interests}

The authors declare no competing interests.

\section{Additional information}

Correspondence and requests for materials should be addressed to M.-Y.H.

Reprints and permissions information is available at www.nature.com/reprints.

Publisher's note Springer Nature remains neutral with regard to jurisdictional claims in published maps and institutional affiliations.

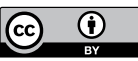

Open Access This article is licensed under a Creative Commons Attribution 4.0 International License, which permits use, sharing, adaptation, distribution and reproduction in any medium or format, as long as you give appropriate credit to the original author(s) and the source, provide a link to the Creative Commons license, and indicate if changes were made. The images or other third party material in this article are included in the article's Creative Commons license, unless indicated otherwise in a credit line to the material. If material is not included in the article's Creative Commons license and your intended use is not permitted by statutory regulation or exceeds the permitted use, you will need to obtain permission directly from the copyright holder. To view a copy of this license, visit http://creativecommons.org/licenses/by/4.0/.

(c) The Author(s) 2019 